\title{
Low-frequency instability of circularly polarized microwave-pulsed-field-produced plasmas*
}

\author{
B. Shokri ${ }^{\dagger}$ \\ Physics Dept. and Laser Research Inst. of Shahid Beheshti University, Evin,Tehran,Iran and \\ Inst. for studies in Theoretical Physics and Mathematics, P.O.Box 19395-3351, Tehran, Iran and \\ Dept. of Electrical and Computer Engineering, University of Alberta, Edmonton, T6G 2V4, Canada \\ M. Ghorbanalilu \\ Physics Department of Shahid Beheshti University, Evin, Tehran, Iran
}

\begin{abstract}
The plasma produced by a high-power pulsed circularly polarized microwave field which is much weaker than the atomic fields is investigated in the non-relativistic limit. It is shown that the resulting electron distribution function is non-equilibrium and anisotropic. Furthermore, the dispersion equations derived to study and analyze low-frequency ion oscillations. It is shown an instability may grow in the aforementioned system only when the ion density exceeds a critical value.
\end{abstract}

\footnotetext{
*PACS No: 52.40.Db,52.35.Hr

${ }^{\dagger}$ E-mail:B-shokri@sbu.ir
} 


\section{INTRODUCTION}

A gas discharge in a microwave (MW) field is a rather complicated phenomenon that exhibits a variety of features. Numerous theoretical and experimental studies have been devoted to this phenomenon. Interest in such a discharge is related not only to its potential for technological applications, but also for general understanding of the discharge physics. ${ }^{1,2}$ A large amount of experimental data has been accumulated and many theoretical studies have been made to explain its various details. A number of experiments have been performed with radiation sources operating at wave lengths of $8.5,4,2$, and $0.8 \mathrm{~cm}^{3}$ The strong MW field interaction with a gas raises interesting questions about the ionization mechanism and the electron distribution function created by this field. Furthermore, the interaction between high-power microwave fields with inhomogeneous plasmas results in a variety of interesting phenomena such as frequency upshift. ${ }^{4}$

In such a strong microwave wave field the electron oscillatory energy $\epsilon_{\mathrm{e}}$ is much higher than the ionization energy $I_{\text {ioniz }}$ of the gas atoms

$$
\epsilon_{\mathrm{e}}=\frac{e^{2} E_{0}^{2}}{2 m \omega_{0}^{2}} \gg I_{\text {ioniz }}
$$

where $\omega_{0}$ is the radiation frequency, $m$ is the electron mass, and $E_{0}$ is the electric field strength. When the field amplitude is comparable to the atomic field strength $E_{\mathrm{a}} \approx 5.1 \times 10^{9} \mathrm{~V} \mathrm{~cm}-1$ tunneling ionization becomes an important mechanism for direct ionization of gas atoms. This effect has been thoroughly studied in previous papers. ${ }^{5-7}$ While condition (1) is satisfied by a large margin, relativistic effects are unimportant since the kinetic energy of electron oscillation in an electromagnetic field is comparable to the electron rest mass energy, for present day pulsed sources. In this case, gas atoms are primarily ionized by the impact of oscillating electrons and ionization is governed by the development of an electron avalanche. Recently it has been shown that discharge plasmas produced by super strong pulsed MW fields are subject to the Weibel instability associated with the anisotropic nature of the electron distribution function. ${ }^{8}$ 
In the present paper, we consider the interaction of circularly polarized MW pulsed fields with frequencies $\omega_{0} \simeq 2 \times 10^{10}-2 \times 10^{11} \mathrm{~s}^{-1}$ with a neutral gas in the non-relativistic regime; in particular we study low frequency ion oscillations and their instabilities. We will consider a pulsed radiation source which is capable of generating radiation with an intensity of about $10^{8} \mathrm{~W} \mathrm{~cm}^{-2}$, whose electric field $E_{0} \leq 10^{6} \mathrm{~V} \mathrm{~cm}^{-1}$ is much weaker than the atomic field $E_{\mathrm{a}}$. In this case, we study the electron distribution function (EDF) and the stability of the discharge plasma in the aforementioned frequency range at non-relativistic electron oscillation energy $\epsilon_{\mathrm{e}}$.

This work is organized in five sections. In Sec. II we review the EDF generated by the interaction of linearly polarized MW fields with a neutral gas. In Sec. III we consider the effect of circularly MW fields on the plasma produced and find the general dispersion equation for low frequency oscillations. In Sec. IV we solve the dispersion equations for low frequencies to find an instability of the system and its growth rate. Finally, a summary and conclusions are presented in Sec. V.

\section{DISTRIBUTION FUNCTION}

Under condition (1) the thermal velocity of the electrons in a discharge plasma can be neglected in comparison to the electron oscillation velocity in the MW radiation field. Since the collision frequency is much smaller than the MW field frequency, we can ignore the collisional randomization of the forced electron oscillation as well. Furthermore, if the plasma density $n_{\mathrm{e}}(t)$ produced by

the field during gas breakdown is less than the critical density (i.e., $\omega_{0}^{2}>\omega_{\text {pe }}^{2}=4 \pi n e^{2} / m$ ) we can neglect the effect of the polarization field. Moreover, the plasma density is assumed to be much less than the neutral gas density $n_{0}$ so that the latter can be considered constant. We also suppose that the field was adiabatically switched on in the infinite past. In addition, we can assume that the MW radiation electric field $\mathbf{E}_{\mathbf{0}}$ is constant during a single field period. Therefore, by assuming that the electric field depends only on time, the kinetic equation for the plasma electrons produced 
in the gas breakdown by a strong pulsed field can be written as follows ${ }^{8}$

$$
\frac{\partial f_{0}}{\partial t}+e \mathbf{E}_{\mathbf{0}} \cdot \frac{\partial f_{0}}{\partial \mathbf{p}}=n_{0} \omega_{\text {ioniz }} \delta(\mathbf{p}),
$$

in the non-relativistic limit. Here $\delta(\mathbf{p})$ is the Dirac delta function, $\omega_{\text {ioniz }}$ is the ionization probability of the gas atoms, and $n_{0}$ is the neutral gas number density. In the case of MW gas breakdown, electron-impact ionization is governed by the ionization probability $\omega_{\text {ioniz. }}$ From Eq. (2) the avalanche ionization parameter $\gamma\left(E_{0}\right)$ can be determined.$^{7-9}$ We can neglect the right-hand side of Eq. (2) in the first approximation and calculate the EDF directly by solving the Vlasov equation under the following condition, ${ }^{7-10}$

$$
\omega_{0} \gg \gamma\left(E_{0}\right), \omega_{\text {ioniz }}
$$

Condition (3) depends strongly on the neutral gas density and is well satisfied at gas pressures of $p_{0} \simeq 10-100$ Torr. In this approximation, to calculate the electron energy distribution function, we assume the field components with circular polarization to be

$$
E_{x}=E_{0} \sin \omega_{0} t, \quad E_{y}=E_{0} \cos \omega_{0} t, \quad E_{z}=0,
$$

where the electric field amplitude $E_{0}$ describes a slowly varying (over the field period) MW pulsed envelope.

Considering condition (3) and solving Eq. (2), by averaging the distribution function over the MW field period, we find the following expression for the $\mathrm{EDF}^{8}$

$$
\left\langle\tilde{f}_{0}\left(v_{\perp}, v_{z}\right)\right\rangle=\frac{\delta\left(v_{z}\right)}{2 \pi^{2} v_{\perp} \sqrt{4 v_{E}^{2}-v_{\perp}^{2}}},
$$

where, $f_{0}(\mathbf{v}, t)=n_{e}(t) \tilde{f}_{0}(\mathbf{v})$ and $v_{E}=e E_{0} / m \omega_{0}$ is the electron oscillatory velocity in an alternating electric field; $v_{z}$ is the perpendicular component of electron velocity with respect to the electric field; $v_{\perp}$ is the component of velocity in the plane of the electric field; $\tilde{f}_{0}(\mathbf{v})$ satisfies the normalization condition $\int d \mathbf{v} \tilde{f}_{0}(\mathbf{v})=1$. 
Since a pulse of a strong MW field actually contains on the order of 10-100 wave periods the EDF is in principle unsteady. However, as one would naturally expect the form of the EDF is determined after several ionizating collisions. Thereafter the electron concentration exponentially increases in time while the shape of the EDF does not change. It is obvious that just on the initial moment of the gas breakdown the EDF is not in equilibrium. But after several periods an EDF is produced such that it usually characterizes the breakdown independent from initial

conditions. Furthermore, the growth rate of the plasma density is expressed by $\sim \gamma / \omega_{0}$. Thus, under the gas low-pressure condition (3) the velocity distribution function of electrons is formed within the field period and is not practically changed furthermore. In addition, during instability development interested in the present paper the plasma density can be considered constant. The rate of collective effects grows with the increase of the plasma particles concentration, and this is why the growth rates of plasma instabilities, usually small compared to the plasma frequency, are proportional to the plasma frequency.

Under conditions (1) and (3) the EDF is well described by Eq. (5) since, at the instant of originating, the electrons are evenly distributed in phase with the wave electric field.

\section{DISPERSION EQUATION}

The theory of the interaction of MW fields with a plasma has been widely developed and covers an increasing range of plasma phenomena. ${ }^{11}$ In Sec. II the distribution function of the charged particles for a homogeneous plasma produced by an external circularly polarized MW electric field defined by Eq. (4) was obtained. The stability of such a plasma can be analyzed by considering small perturbations of this distribution. To begin with, we analyze MW fields with frequencies higher than all characteristic plasma frequencies

$$
\omega_{0} \gg \omega_{\mathrm{p} \alpha}, \nu_{\alpha}
$$


where $\omega_{\mathrm{p} \alpha}, \nu_{\alpha}$ are the plasma frequency, and collision frequency for $\alpha$ species (ions or electrons), respectively. To first-order approximation, the plasma in a MW field may be regarded as isotropic. Consequently, the field $\mathbf{E}_{\mathbf{0}}(t)$ obeys the dispersion equation for transverse wave

$$
k_{0}=\frac{\omega_{0}}{c} \sqrt{1-\frac{\omega_{p e}^{2}}{\omega_{0}^{2}}} \simeq \frac{\omega_{0}}{c} .
$$

where $c$ is the speed of light in vacuum. Therefore, the MW field can be considered homogeneous for processes with a characteristic length of inhomogeneity $k^{-1}$ (where $k^{-1}$ is much smaller than $k_{0}^{-1}$, or $\left.k \gg \omega_{0} / c\right)$. Assuming this condition, we analyze quasi-longitudinal oscillations in the stability of the MW-field-produced plasma. In reality, the oscillation frequencies are of the order of characteristic plasma frequencies, i.e., $\omega \sim \omega_{\text {pe. }}$ Taking into account relation $(7)$, we find the validity condition of the quasi-longitudinal approximation to be $\omega \ll \omega_{0} \simeq k_{0} c \ll k c$.

The MW electric field with circular polarization primarily interacts with the neutral gas and produces a plasma whose EDF and properties were summarized in Sec. II. Since we will study instabilities whose growth rates are higher than the ionization rate we can assume a constant electron density. It is clear that, for relatively weak fields in real plasmas, electrons are accelerated to velocities significantly exceeding their thermal velocity during the period of oscillatory electric field and the mean free time. The result is a state in which electrons are moving with respect to motionless ions. Such a charged particle beam is unstable and its growth increment is rather large, of the order of the plasma ion frequency or even larger. In this section we derive the general dispersion relation for the MW produced plasma in the limit of low-frequency ion oscillation perturbations. Since the effect of a high frequency electric field on the plasma ions can be neglected compared to its effect on the electrons, the ion velocity distribution function can be regarded as isotropic (usually Maxwellian) and the EDF is given by Eq. (5).

Linearizing the Vlasov equations for electrons and ions in a collisionless plasma we obtain the 
following equations

$$
\begin{gathered}
\frac{\partial \delta f_{\mathrm{e}}}{\partial t}+\imath \mathbf{k} \cdot \mathbf{v} \delta f_{\mathrm{e}}+\frac{e E_{0}}{m}\left(\hat{\mathbf{i}} \sin \omega_{0} t+\hat{\mathbf{j}} \cos \omega_{0} t\right) \cdot \frac{\partial \delta f_{\mathrm{e}}}{\partial \mathbf{v}}+\frac{e \mathbf{E}}{m} \cdot \frac{\partial f_{0 \mathrm{e}}\left(\mathbf{p}-\mathbf{p}_{\mathbf{0}}\right)}{\partial \mathbf{v}}=0 \\
\frac{\partial \delta f_{\mathrm{i}}}{\partial t}+\imath \mathbf{k} \cdot \mathbf{v} \delta f_{\mathrm{i}}+\frac{e_{\mathrm{i}} \mathbf{E}}{M} \cdot \frac{\partial f_{0 \mathrm{i}}(\mathbf{p})}{\partial \mathbf{v}}=0
\end{gathered}
$$

for small deviations from the zero-order distributions. Here, $\hat{\mathbf{i}}$ and $\hat{\mathbf{j}}$ are unit vectors along $\mathrm{x}$ and y direction, respectively; $f_{0 \mathrm{e}}\left(\mathbf{p}-\mathbf{p}_{\mathbf{0}}\right)$ and $f_{0 \mathrm{i}}(\mathbf{p})$ are the zero-order distribution functions of the electrons and the ions, respectively; $e, m, e_{\mathrm{i}}$, and $M$ are the electron and ion charge and mass, respectively and $\mathbf{p}_{\mathbf{0}}=e \int^{t} \mathbf{E}_{\mathbf{0}} d t=-e \mathbf{E}_{\mathbf{0}} \cos \omega_{0} t / \omega_{0}$ and $\imath=\sqrt{-1}$. The small perturbations $\delta f_{\mathrm{e}}$ and $\delta f_{\mathrm{i}}$ depend on the coordinates in the form of $\exp (\imath \mathbf{k} \cdot \mathbf{x})$, because of the assumption of homogeneity; $\mathbf{E}=-\nabla \phi$ is the electric field due to the perturbations satisfying the Poisson equation

$$
k^{2} \phi=4 \pi e \int d \mathbf{p} \delta f_{\mathrm{e}}+4 \pi e_{\mathrm{i}} \int d \mathbf{p} \delta f_{\mathrm{i}} .
$$

In order to solve Eqs. (8)-(10) it is convenient to introduce a new function

$$
\psi_{\mathrm{e}}(\mathbf{p}, t)=\exp \left\{-\frac{\imath e E_{0}}{m \omega_{0}^{2}}\left(k_{x} \sin \omega_{0} t+k_{y} \cos \omega_{0} t\right)\right\} f_{\mathrm{e}}\left(\mathbf{p}+\frac{e E_{0}}{\omega_{0}}\left[\mathbf{i} \cos \omega_{0} t-\mathbf{j} \sin \omega_{0} t\right]\right),
$$

where $k_{x}$ and $k_{y}$ are the component of the wave vector $\mathbf{k}$. The system of equations then takes the form

$$
\begin{gathered}
\quad \frac{\partial \psi_{\mathrm{e}}}{\partial t}+\imath \mathbf{k} \cdot \mathbf{v} \psi_{\mathrm{e}}-\imath \mathbf{k} \cdot \frac{\partial f_{0 \mathrm{e}}(\mathbf{p})}{\partial \mathbf{v}} \frac{4 \pi e}{m k^{2}} \\
\times\left[e \int d \mathbf{p} \psi_{\mathrm{e}}(\mathbf{p})+e_{\mathrm{i}} \int d \mathbf{p} \delta f_{\mathrm{i}}(\mathbf{p}) \exp \left\{-\frac{\imath e E_{0}}{m \omega_{0}^{2}}\left(k_{x} \sin \omega_{0} t+k_{y} \cos \omega_{0} t\right)\right\}\right]=0, \\
\times \frac{\partial \delta f_{\mathrm{i}}}{\partial t}+\imath \mathbf{k} \cdot \mathbf{v} \delta f_{\mathrm{i}}-\imath \mathbf{k} \cdot \frac{\partial f_{0 \mathrm{i}}(\mathbf{p})}{\partial \mathbf{v}} \frac{4 \pi e_{\mathrm{i}}}{M k^{2}} \\
\times\left[e_{\mathrm{i}} \int d \mathbf{p} \delta f_{\mathrm{i}}+e \int d \mathbf{p} \psi_{\mathrm{e}} \exp \left\{-\frac{\imath e E_{0}}{m \omega_{0}^{2}}\left(k_{x} \sin \omega_{0} t+k_{y} \cos \omega_{0} t\right)\right\}\right]=0 .
\end{gathered}
$$

Applying the expansion

$$
\begin{gathered}
\exp \left\{ \pm \imath\left(k_{x} r_{E} \sin \omega_{0} t+k_{y} r_{E} \cos \omega_{0} t\right)\right\}= \\
\sum_{p=-\infty}^{+\infty} \sum_{m=-\infty}^{+\infty} \exp \left\{ \pm \imath(p+m) \omega_{0} t\right\} \quad \exp \left\{ \pm \imath \frac{m \pi}{2}\right\} J_{p}\left(k_{x} r_{E}\right) J_{m}\left(k_{y} r_{E}\right)
\end{gathered}
$$


and introducing the decomposition

$$
\left(\begin{array}{c}
\psi_{\mathrm{e}} \\
\delta f_{\mathrm{i}}
\end{array}\right)=\exp (-\imath \omega t) \sum_{l=-\infty}^{+\infty} \sum_{n=-\infty}^{+\infty} \exp \left\{ \pm \imath(l+n) \omega_{0} t\right\}\left(\begin{array}{c}
\psi_{\mathrm{e}_{l n}} \\
\delta f_{\mathrm{i}_{l n}}
\end{array}\right)
$$

we find from Eq. (12) the following equations

$$
\begin{aligned}
& -\imath\left[\omega+(l+n) \omega_{0}\right] \psi_{\mathrm{e}_{l n}}+\imath \mathbf{k} \cdot \mathbf{v} \psi_{\mathrm{e}_{l n}}-\imath \mathbf{k} \cdot \frac{\partial f_{0 \mathrm{e}}}{\partial \mathbf{v}} \frac{4 \pi e}{m k^{2}}
\end{aligned}
$$

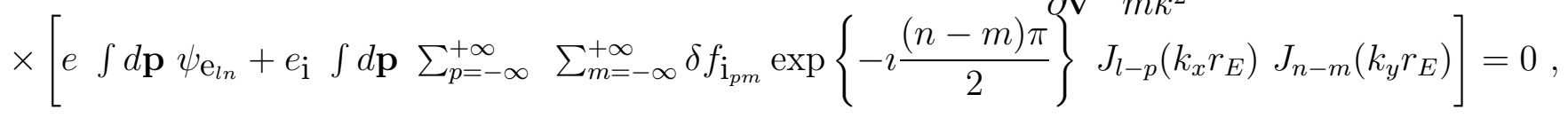

$$
\begin{aligned}
& -\imath\left[\omega+(l+n) \omega_{0}\right] \delta f_{\mathrm{i}_{l n}}+\imath \mathbf{k} \cdot \mathbf{v} \delta f_{\mathrm{i}_{l n}}-\imath \mathbf{k} \cdot \frac{\partial f_{0 \mathbf{i}}}{\partial \mathbf{v}} \frac{4 \pi e_{\mathbf{i}}}{M k^{2}} \\
& \times\left[e_{\mathrm{i}} \int d \mathbf{p} \delta f_{\mathrm{i}_{l n}}+e \int d \mathbf{p} \sum_{p=-\infty}^{+\infty} \sum_{m=-\infty}^{+\infty} \psi_{\mathrm{e}_{l n}} \exp \left\{\imath \frac{(m-n) \pi}{2}\right\} J_{p-l}\left(k_{x} r_{E}\right) J_{m-n}\left(k_{y} r_{E}\right)\right]=0 \text {, }
\end{aligned}
$$

where $\mathbf{r}_{E}=e \mathbf{E}_{\mathbf{0}} / m \omega_{0}^{2}$ is the amplitude of the electron oscillation in the MW electric field and $J_{n}$ is the Bessel function of order $n$.

Now using the notation

$$
u_{\mathrm{e}_{l n}}=e \int d \mathbf{p} \psi_{\mathrm{e}_{l n}}, \quad u_{\mathrm{i}_{l n}}=e_{\mathrm{i}} \int d \mathbf{p} \delta f_{\mathrm{i}_{l n}}
$$

we can write the formal solution of Eq. (15) in the form of a coupled set of equation, as follows:

$$
\begin{gathered}
u_{\mathrm{e}_{l n}}=-\delta \varepsilon_{\mathrm{e}}\left(\omega+(l+n) \omega_{0}, \mathbf{k}\right) \times \\
{\left[u_{\mathrm{e}_{l n}}+\sum_{p=-\infty}^{+\infty} \sum_{m=-\infty}^{+\infty} u_{\mathrm{i}_{p m}} \exp \left\{\imath \frac{(m-n) \pi}{2}\right\} J_{p-l}\left(k_{x} r_{E}\right) J_{m-n}\left(k_{y} r_{E}\right)\right],} \\
u_{\mathrm{i}_{l n}}=-\delta \varepsilon_{\mathrm{i}}\left(\omega+(l+n) \omega_{0}, \mathbf{k}\right) \times \\
{\left[u_{\mathrm{i}_{l n}}+\sum_{p=-\infty}^{+\infty} \sum_{m=-\infty}^{+\infty} u_{\mathrm{e}_{p m}} \exp \left\{-\imath \frac{(n-m) \pi}{2}\right\} J_{l-p}\left(k_{x} r_{E}\right) J_{n-m}\left(k_{y} r_{E}\right)\right],}
\end{gathered}
$$

where $\delta \varepsilon_{\mathrm{e}}(\omega, \mathbf{k})$ and $\delta \varepsilon_{\mathrm{i}}(\omega, \mathbf{k})$ are the partial contributions of the electron and ion susceptibilities to the longitudinal dielectric permittivity ${ }^{8}$

$$
\varepsilon(\omega, \mathbf{k})=1+\delta \varepsilon_{\mathrm{i}}+\delta \varepsilon_{\mathrm{e}}
$$


The existence of solutions to this infinite set of coupled equations gives the dispersion equation for small-amplitude longitudinal oscillations in the external MW electric field. Since it is a determinant of infinite order, it is impossible to analyze in general. However, for the most interesting limiting cases this determinant can be simplified and the oscillation spectrum can be found. For example, all the values $\delta \varepsilon_{\mathrm{e}, \mathrm{i}}\left(\omega+n \omega_{0}, \mathbf{k}\right)$ with $n \neq 0$, are small compared to unity in the very high frequency limit, when condition (6) is satisfied. Assuming this limit, only $u_{\mathrm{e}_{00}}$ and $u_{\mathrm{i}_{00}}$ are nonzero in Eq. (16); thus, we obtain

$$
\begin{aligned}
& {\left[1+\delta \varepsilon_{\mathrm{e}}(\omega, \mathbf{k})\right] u_{\mathrm{e}_{00}}+\delta \varepsilon_{\mathrm{e}}(\omega, \mathbf{k}) J_{0}\left(k_{x} r_{E}\right) J_{0}\left(k_{y} r_{E}\right) u_{\mathrm{i}_{00}}=0} \\
& J_{0}\left(k_{x} r_{E}\right) J_{0}\left(k_{y} r_{E}\right) \delta \varepsilon_{\mathrm{i}}(\omega, \mathbf{k}) u_{\mathrm{e}_{00}}+\left[1+\delta \varepsilon_{\mathrm{i}}(\omega, \mathbf{k})\right] u_{\mathrm{i}_{00}}=0,
\end{aligned}
$$

which gives the following dispersion equation

$$
1+\delta \varepsilon_{\mathrm{e}}(\omega, \mathbf{k})+\delta \varepsilon_{\mathrm{i}}(\omega, \mathbf{k})+\delta \varepsilon_{\mathrm{e}}(\omega, \mathbf{k}) \delta \varepsilon_{\mathrm{i}}(\omega, \mathbf{k})\left[1-J_{0}^{2}\left(k_{x} r_{E}\right) J_{0}^{2}\left(k_{y} r_{E}\right)\right]=0
$$

By deriving Eq. (18), we fully neglected particle collisions. However, this equation is also valid for collisional plasmas if the oscillation velocity component of the particles is small compared to the thermal velocity. The external MW field has no effect on the collision process, i.e., on the scattering cross sections.

\section{INSTABILITY OF THE MW PRODUCED PLASMA.}

The instability analyzed in this section occurs in the range $\omega_{0} \gg k v_{E} \approx \omega_{\text {pe. }}$ It only occurs for a positive (non-equilibrium) slope in the EDF (5) and never for an equilibrium (Maxwellian) electron energy distribution in this frequency range. Taking a Maxwell distribution function for cold ions and the distribution function (5) for electrons, the electron and ion susceptibilities $\delta \varepsilon_{\mathrm{e}, \mathrm{i}}(\omega, \mathbf{k})$ 
are given by ${ }^{8,11}$

$$
\begin{gathered}
\delta \varepsilon_{\mathrm{i}}(\omega, k)=-\frac{\omega_{\mathrm{pi}}^{2}}{\omega^{2}}, \\
\delta \varepsilon_{\mathrm{e}}(\omega, \mathbf{k})=\frac{\omega_{\mathrm{pe}}^{2}}{k^{2}} \int d v \frac{k \partial \tilde{f}_{0}(v, t) / \partial v}{\omega-k v}=-\frac{\omega_{\mathrm{pe}}^{2} k_{\perp}^{2}}{\omega^{2} k^{2}} \frac{s}{\sqrt{1-\frac{4 k_{\perp}^{2} v_{E}^{2}}{\omega^{2}}}}-\frac{\omega_{\mathrm{pe}}^{2} s}{\omega^{2} \sqrt{\left(1-\frac{4 k_{\perp}^{2} v_{E}^{2}}{\omega^{2}}\right)^{3}}},
\end{gathered}
$$

where

$$
s=\left\{\begin{array}{ccr}
k_{\perp}=\sqrt{k_{x}^{2}+k_{y}^{2}}, \\
1 & \text { for } & \operatorname{Re}(\omega) \neq 0 \\
1 / 2 & \text { for } & \operatorname{Re}(\omega)=0
\end{array}\right\} \text { when } \quad \frac{4 k_{\perp}^{2} v_{E}^{2}}{\omega^{2}}<1,
$$

where $\operatorname{Re}(\omega)$ and $\operatorname{Im}(\omega)$ are the real and imaginary part of $\omega$, respectively. Substituting expressions (19) and (20) into the dispersion equation (18), we obtain the following dispersion relations

$$
\begin{cases}\omega^{2}=\omega_{\mathrm{pi}}^{2}\left[1+\imath\left(\frac{\omega_{\mathrm{pe}}^{2} \omega_{\mathrm{pi}}}{\left|2 k_{\perp} v_{E}\right|^{3}}-\frac{\omega_{\mathrm{pe}}^{2} k_{\perp}^{2}}{\omega_{\mathrm{pi}}\left|2 k_{\perp} v_{E}\right| k^{2}}\right) J_{0}^{2}\left(\frac{k_{\perp} v_{E}}{\omega_{0}}\right)\right] & 4 k_{\perp}^{2} v_{E}^{2} \gg \omega_{\mathrm{pe}}^{2}, \\ \omega=\frac{\left|2 k_{\perp} v_{E}\right| k_{\perp}}{k}\left[1-\frac{\imath}{2}\left(\frac{\left|2 k_{\perp} v_{E}\right|^{2} k}{\omega_{\mathrm{pe}}^{2} k_{\perp}}-\frac{\omega_{\mathrm{pi}}^{2} k^{3}}{\omega_{\mathrm{pe}}^{2} k_{\perp}^{3}}\right)\right] & 4 k_{\perp}^{2} v_{E}^{2} \ll \omega_{\mathrm{pe}}^{2}\end{cases}
$$

corresponding to unstable low frequency ion oscillations. Here, the following approximation

$$
J_{0}^{2}\left(\frac{k_{\perp} v_{E}}{\omega_{0}}\right) \approx J_{0}^{2}\left(\frac{k_{x} v_{E}}{\omega_{0}}\right) J_{0}^{2}\left(\frac{k_{y} v_{E}}{\omega_{0}}\right),
$$

was used. Equation (21) show that in the case of a circularly polarized MW field the low frequency oscillation takes place only for perturbations satisfying the following condition:

$$
\frac{\omega_{\mathrm{pi}}^{2}}{\left|2 k_{\perp} v_{E}\right|^{2}} \geq \frac{k_{\perp}^{2}}{k^{2}} .
$$


This instability resulting from the positive slope of the distribution function (5) can be interpreted as the stimulated Cherenkov excitation of the low frequency ion oscillations by the radiation field of the oscillating electrons. Perturbations that do not satisfy the condition (23) are not unstable. Of course, according to approximations and assumption used, the instability satisfying condition (23) can only occur when the growth rate exceeds the ionization rate, i.e., the growth rate should exceed the avalanche ionization constant $\gamma\left(E_{0}\right)$.

\section{CONCLUSION}

In analyzing the interaction of a high frequency circularly polarized MW field with a neutral gas, we obtained the general dispersion relation for low frequency waves in the MW produced plasma. From Eq. (23) for the instability condition it is necessary that the the ion density exceeds the critical value $n_{i} \geq M k_{\perp}^{4} E_{0}^{2} / \pi m^{2} k^{2} \omega_{0}^{2}$. Under such condition the electrons transfer electric field energy to the ions and stimulated Cherenkov excitation of low frequency ion oscillations takes place. This effect requires a positive slope in the distribution function and, in this case, the

instability can propagate when the grow rate exceeds the avalanche ionization rate $\gamma\left(E_{0}\right)$. Other work underway, is concerned with the case of linearly polarizd MW fields. 


\section{References}

[1] G. A. Askar'yan, G. M. Batanov, A. E. Barkhudarov, et al., J. phys. D: Appl. phys., 27, 1311 (1994).

[2] L. p. Grachev, I. I. Esakov, K. V. Khodataev, and V. V. Tsyplenkov, Fiz. Plasmy, 18, 411, (1992) [Sov. J. Plasma Phys. (Engl. transl. ), 18, 3, 216 (1992)].

[3] A. J. Taylor, C. R. Tallman, J. P. Roberts, et al., Opt. Lett., 15, 39 (1990).

[4] X. Xu, N. Yugami, and Y. Nishida, Phys. Rev. E, 55, Issu 3, 3328-3332, (1997).

[5] L. V. Keldish, Zh. Éksp. Teor. Fiz., 47, 1945, (1964) [Sov. Phys. JETP 20, 1307, (1964)].

[6] A. M. Perelomov, V. S. Popov, and, M. V. Terent'ev, Zh. Éksp. Teor. Fiz., 50, 1393, (1966), [Sov. Phys. JETP, 23, 924, (1966)].

[7] M. V. Kuzelev, and A. A. Rukhadze, Fiz. plazmy, 27, No. 2, 165, (2001), [Sov. J. Plasma Phys. (Engl. transl.), 27, No. 2, 153, (2001)].

[8] B. Shokri, M. Ghorbanalilu, Phys. of Plasmas, 11, 2989, (2004).

[9] V. Yu. Bychenkov, and V.T. Tikhonchuk, Laser Focus 2, 525, (1992).

[10] A. A. Rukhadze, K. F. Sergeichev, Phys. Lett. A, 288, 316, (2001).

[11] A.F. Alexandrov, L.S. Bogdankevich, and A.A. Rukhadzeh, Principles of Plasma Electrodynamics, (Springer-Verlag, Heidelberg, 1984). 